\title{
Associações entre gênero e desenvolvimento linguístico em crianças de uma creche pública
}

\author{
Associations between gender and language development in children at a public daycare center \\ Asociaciones entre género y desarrollo del lenguaje en los niños de una guardería pública
}

Recebido: 17/05/2021 | Revisado: 27/05/2021 | Aceito: 11/06/2021 | Publicado: 24/06/202

\author{
Gabriela da Silva Leandro \\ ORCID: https://orcid.org/0000-0001-8188-3383 \\ Universidade do Estado do Pará, Brasil \\ E-mail: gabrielaleandro48@gmail.com \\ Adriele Francisca da Silva Souza \\ ORCID: https://orcid.org/0000-0003-2511-2490 \\ Universidade do Estado do Pará, Brasil \\ E-mail: adriele.fsouza@ aluno.uepa.br \\ Ivete Furtado Ribeiro Caldas \\ ORCID: https://orcid.org/0000-0002-2095-101X \\ Universidade do Estado do Pará, Brasil \\ E-mail: ivbeiro@yahoo.com.br \\ Elson Ferreira Costa \\ ORCID: https://orcid.org/0000-0003-4115-9029 \\ Universidade Federal do Pará, Brasil \\ E-mail: elsonfcosta@gmail.com
}

\begin{abstract}
Resumo
O desenvolvimento infantil contempla quatro grandes áreas: motor grosseiro, motor fino-adaptativo, pessoal-social e linguagem, sendo todas influenciadas por caracteres intrínsecos e extrínsecos. A linguagem é um instrumento social utilizado em interações comunicativas e diversos fatores podem influenciar nesse processo, como o gênero. Os estímulos ofertados a meninos e meninas são distintos, baseados em diferenças socioculturais instituídas ao longo dos séculos. Objetivo: Avaliar a associação entre o gênero e o desenvolvimento linguístico em crianças de uma creche pública no município de Marabá (PA). Método: Trata-se de um estudo transversal, realizado em 62 crianças, com idade entre 3 a 6 anos. O intrumento utilizado foram o Questionário socioeconômico e o Teste de Triagem de Desenvolvimento de Denver II. Foi utilizado o Teste U (Teste não paramétrico de Mann Whitney) com nível de significância de 5\%. Resultados: As meninas obtiveram maiores médias de acertos, destacando-se: compreende 3 adjetivos $(0.94 \pm 0.25)$, reconhece 4 ações $(0.94 \pm 0.25)$ e define 3 objetos pelo uso $(0.97 \pm 0.18)$. Conclusão: Crianças do sexo feminino possuem desfecho mais favorável no processo de aquisição da fala comparado ao sexo masculino.
\end{abstract}

Palavras-chave: Desenvolvimento infantil; Crianças; Construção social de gênero; Neurobiologia.

\begin{abstract}
The incentives offered to boys and girls are different, based on socio-cultural differences instituted over the centuries. Objective: To analyze the language development of children in a public daycare center and to verify possible associations of the linguistic outcome with difference between genders. Method: This is a cross-sectional study, carried out on 62 children, aged 3 to 6 years. The instrument used was the Denver II Development Screening Test. To compare the mean scores, the Mann Whitney nonparametric test was used, with a significance level of 5\% ( $\mathrm{p}<0.05$ ). Results: The association of the Denver II outcome with gender showed that the girls obtained higher average scores, highlighting: it comprises 3 adjectives $(0.94 \pm 0.25)$, recognizes 4 actions $(0.94 \pm 0.25)$ and defines 3 objects for use $(0.97 \pm 0.18)$. Conclusion: Female children have a more favorable outcome in the speech acquisition process compared to men.
\end{abstract}

Keywords: Child development; Children; Gender social construction; Neurobiology.

\section{Resumen}

Los incentivos que se ofrecen a niños y niñas son diferentes, basados en las diferencias socioculturales instituidas a lo largo de los siglos. Objetivo: Analizar el desarrollo del lenguaje de niños en una guardería pública y verificar posibles asociaciones del resultado lingüístico con diferencia entre géneros. Método: Se trata de un estudio transversal, realizado en 62 niños de 3 a 6 años. El instrumento utilizado fue el Prueba de detección de desarrollo Denver II. Se utilizó la prueba no paramétrica de Mann Whitney para comparar las puntuaciones medias, con un nivel de significancia del 5\% ( $\mathrm{p}<0,05)$. Resultados: La asociación del desenlace Denver II con el género mostró que las niñas obtuvieron puntajes promedio más altos, destacando: comprende 3 adjetivos $(0.94 \pm 0.25)$, reconoce 4 acciones $(0.94$ 
$\pm 0.25)$ y define 3 objetos por uso $(0,97 \pm 0,18)$. Conclusión: las niñas tienen un resultado más favorable en el proceso de adquisición del habla en comparación con los hombres.

Palabras clave: Desarrollo infantil; Infancia; Construcción social de género; Neurobiología.

\section{Introdução}

O desenvolvimento humano é caracterizado pela capacidade contínua do indivíduo em se adaptar ao ambiente em que está inserido. Pode ser definido como um processo único, contínuo, dinâmico e progressivo. É marcado por alterações físicas, cognitivas, comportamentais, neurológicas e sociais, que se expressam de forma gradual à medida que o indivíduo atinge a competência de corresponder às suas necessidades e as do ambiente. O desenvolvimento infantil contempla quatro grandes áreas: motor grosseiro, motor fino-adaptativo, pessoal-social e linguagem, sendo todas influenciadas por caracteres intrínsecos e extrínsecos (Costa; Cavalcante, \& Dell'aglio, 2015). Essa última área, merece destaque, pois envolve o reconhecimento, a compreensão e a utilização do código linguístico, através da interação de múltiplos fatores genéticos, ambientais e psicossociais (Pinto, Isotani, Sabatés \& Perissinoto, 2015).

A linguagem é um instrumento social utilizado em interações comunicativas, definida como um sistema convencional de símbolos que são entrelaçados de modo sistemático visando o armazenamento e a troca de informações, sendo sua aquisição um processo gradativo, demarcado pela faixa etária, em que as aquisições de cada fase servem como base e impulso para a próxima (Mélo, Lucchesi, Junior, \& Signorelli, 2020). O período de maior suscetibilidade ao desenvolvimento linguístico é durante a primeira infância, 0 a 5 anos, fase determinante e imprescindível para a formação do indivíduo (Araújo, 2017).

O processo de aquisição linguística envolve a conexão entre áreas cerebrais distintas que compõem a zona de linguagem, a qual em 90\% dos indivíduos se localiza no hemisfério cerebral esquerdo. Duas regiões são primordiais, a área de Broca, região pré-motora do lobo frontal, responsável pela articulação motora para a execução da fala, e a área de Wernicke, situada na região posterior da circunvolução temporal superior, composta por fibras sensoriais, responsável pelo processamento e compreensão da linguagem (Duarte, Vasconcelos, \& Batalha, 2011).

O desenvolvimento linguístico é dependente, por um lado, de fatores anatomofuncionais e, por outro, de estímulos verbais provenientes do ambiente. Diversos fatores podem influenciar o processo de aquisição linguística, podendo trazer grandes repercussões a curto, médio e longo prazo na vida da criança. Dentre eles ressaltam-se os fatores socioeconômicos, nutricionais, socio-familiares e a diferença de gêneros (Passaglio et al., 2015).

Destacando-se o tipo de gênero, pesquisas apontam diferenças em relação ao processo do desenvolvimento linguístico. As meninas estão à frente cerca de 1 mês no número de palavras que falam em relação aos meninos, próximo aos 22 meses, produzem em média 300 palavras diferentes; enquanto os meninos alcançam essa marca por volta dos 23 ou 24 meses. Em torno de 2 anos e meio, as meninas possuem a capacidade de juntar cerca de oito palavras por vez; os meninos, somente cinco; além do que as frases das meninas tendem a ser mais complexas e longas (Eliot, 2013). Essas diferenças podem ser justificadas pela presença de hormônios nas crianças do sexo feminino, como os esteroides, importantes reguladores do comportamento vocal em relação à linguagem, os quais ocorrem durante um pico hormonal transitório denominado "minipuberdade" (Wermke et al., 2018). Outro estudo também aponta diferença na cronologia da mielinização em áreas cerebrais responsáveis pela linguagem, ocorrendo de forma mais precoce em meninas (Pinheiro, 2007).

Além do mais, merece atenção a disparidade quanto aos estímulos dispensados às crianças baseados em diferenças socioculturais instituídas ao longo dos séculos. Aos meninos são atribuídas características de intrepidez, frieza e heroísmo, sendo incentivados de forma precoce a brincadeiras agitadas e vigorosas que estimulam mais intensamente a área motora ampla. Já as meninas são rotuladas como seres delicados, sensíveis e mais sociáveis, recebendo, assim, maior estímulo às áreas motor fino-adaptativo e linguagem (Raffaini, 2018). Essa assimetria torna-se evidente principalmente no âmbito escolar e familiar (Ciribelli, \& Rasera, 2019). 
Um estudo brasileiro voltado para a avaliação de alterações fonológicas na infância, demonstrou que 68,3\% dos distúrbios da comunicação predominavam no gênero masculino, sendo mais vulnerável a falhas no processo de desenvolvimento cognitivo e de linguagem, perceptíveis desde o primeiro ano de vida (Longo et al., 2017). Embora os meninos demonstrem leve desvantagem no desenvolvimento linguístico, as oportunidades de melhora das habilidades verbais são inúmeras, em virtude da neuroplasticidade cerebral, a qual permite a mudança estrutural e funcional dos circuitos neuronais em resposta aos estímulos ambientais e favorece a aquisição de novas aprendizagens (Li, Legault, \& Litcofsky, 2014; Guerim, 2020).

As interações ricas em linguagem, por meio da fala e de leituras compartilhadas, contribuem de forma positiva para a promoção do bem-estar físico, social, emocional e cognitivo, favorecendo o desenvolvimento linguístico apropriado (Zauche, Thul, Mahoney, \& Stapel-Wax, 2016; Costa et al., 2019).

Dessa forma, o tipo de gênero da criança pode ter relação direta sobre o desenvolvimento linguístico, uma vez que oportunidades fornecidas à criança e as suas expectativas sociais influenciam diretamente na capacidade de aprendizagem e de socialização. Nesse sentido, objetiva-se avaliar a associação entre o gênero e o desenvolvimento linguístico em crianças de uma creche pública no município de Marabá (PA).

\section{Metodologia}

Trata-se de um estudo transversal com amostra por conveniência. Foram avaliadas 62 crianças, com idade entre 3 a 6 anos, e um responsável de cada criança. A pesquisa foi realizada entre os meses abril a dezembro de 2019, no Núcleo de Educação Infantil (NEI) Maria da Conceição Silva Pereira, localizado no município de Marabá, Pará. NEI`s, são instituições governamentais que promovem educação básica para crianças entre 3 a 6 anos. Esta pesquisa foi aprovada pelo Comitê de Ética em Pesquisa (Parecer nº 3.278.934).

Foram incluídas aquelas crianças regularmente matriculadas e cujos pais e/ou responsáveis aceitaram assinar o Termo de Consentimento Livre e Esclarecido (TCLE). E excluídas aquelas que apresentaram diagnósticos ou suspeitas de quaisquer tipos de distúrbios que afetassem a expressão da fala, alterações auditivas e/ou visuais, comprometimento do sistema nervoso central (SNC), complicações nutricionais e/ou endócrinas.

Para coleta de dados, utilizaram-se os seguintes instrumentos:

Questionário socioeconômico: Utilizado para coletar informações a partir dos pais e/ou responsáveis das crianças. Esse instrumento foi traduzido e adaptado no Brasil por Issler e Giugliani (1997) e composto por 13 itens: 1. Número de pessoas que comem e dormem na casa; 2. Abandono do pai/mãe; 3. Escolaridade dos pais; 4. Atividade dos pais; 5. Relação com o domicílio; 6. Tipo de casa; 7. Número de pessoas que dormem na casa versus lugares para dormir; 8. Abastecimento de água; 9. Deposição de excreta; 10. Coleta de lixo; 11. Energia elétrica; 12. Cozinha independente; 13. Eletrodomésticos do domicílio. A variação de pontuação de cada item possui uma escala de 0 a 4 pontos, cuja menor pontuação total obtida será de 7 pontos e maior de 52 (Costa; Cavalcante, \& Dell'aglio, 2015).

Teste de Triagem de Desenvolvimento de Denver II (Denver II): Representa um teste de triagem, e não um teste diagnóstico. Tem como objetivo avaliar o desenvolvimento das crianças do nascimento aos 6 anos, é composta por 125 itens distribuídos nas seguintes categorias: pessoal-social, motor fino-adaptativo, linguagem e motor grosso. Nesse estudo foi avaliado apenas a categoria linguagem. Em cada uma das habilidades avaliadas foram consideradas três possibilidades de pontuações dos itens que são: passa $(\mathrm{P})$, falha $(\mathrm{F})$, recusa (R) ou sem oportunidade (SO). Ao final de cada avaliação, as crianças foram classificadas conforme seu desempenho: normal, cautela ou atraso (Pinto et al., 2015). Após essa interpretação o desfecho foi classificado em normal, questionável ou atraso para facilitar a análise dos dados.

Antecedendo uma semana para o início das coletas foi enviado um convite para os pais e/ou responsáveis das 
crianças, solicitando a presença na reunião com os pesquisadores para esclarecimentos dos objetivos e as características gerais da pesquisa. Já no primeiro contato foi entregue aos pais e/ou responsáveis o TCLE, aqueles que aceitaram a participação eram encaminhados para coleta de dados juntamente com seus filhos.

Um cronograma foi estabelecido para as avaliações que ocorreram em uma sala reservada nas dependências do NEI que possuía $42 \mathrm{~m}^{2}$ e era bastante arejada e confortável. A sala possuía uma mesa e três cadeiras para acomodar os envolvidos na coleta, além de uma balança mecânica, fita-métrica, piso infantil em forma de quebra-cabeças e brinquedos que foram selecionados dependendo da idade da criança.

Cada sessão teve duração total de trinta (30) minutos e ocorreu no turno matutino e/ou vespertino, sempre respeitando o horário as atividades escolares. Nos primeiros dez (10) minutos iniciais foram coletadas as informações no questionário socioeconômico. Nos vinte (20) minutos seguintes foi realizada a avaliação do desenvolvimento da criança através do Teste de Triagem de Desenvolvimento Denver II (Denver II).

Os dados foram tabulados no programa Microsoft Excel ${ }^{\circledR}$ e analisados no software Statistical Package for Social Sciences (SPSS) versão 22.0. Foram realizadas análises estatísticas descritivas e inferenciais para comparação das médias dos itens. Ademais, para comparar as médias dos escores do Denver II, foi utilizado o Teste U (Teste não paramétrico de Mann Whitney) com nível de significância de 5\% ( $p$ valor $<0,05$ ).

\section{Resultados}

Nossos resultados mostram que em relação às características socioeconômicas das crianças do NEI, 32 (51.62\%) eram do sexo feminino e 30 (48.38\%) do masculino, com média de idade de 61.60 meses. Quanto ao nível de pobreza, 55 (88.70\%) apresentavam renda baixa superior, 4 (6.45\%) renda baixa inferior e $3(4.85 \%)$ miséria. Na avaliação do Teste de Triagem de Desenvolvimento de Denver II, 34 (55.83\%) apresentaram desenvolvimento da linguagem normal, 15 (24.20\%) com atraso e $13(20.97 \%)$ com resultado cautela. Para facilitar o entendimento das análises de dados, as crianças com atraso e com cautela foram agrupadas em um único grupo, nomeado questionável 28 (48,38\%) (Figura 1).

Figura 1: Porcentagem referente ao desfecho do desenvolvimento linguístico segundo o Teste de Triagem de Denver II.

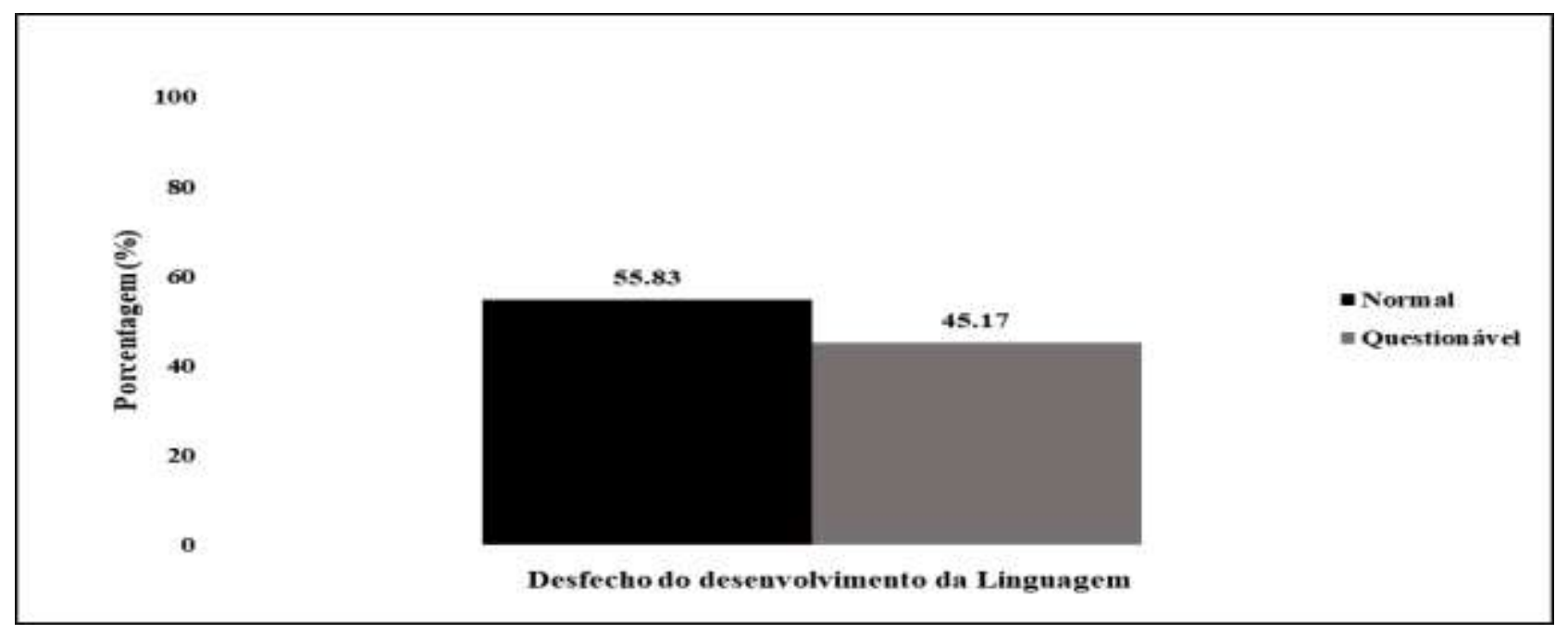

Fonte: Autores (2021).

Em relação às médias das pontuações dos itens avaliados pelo Denver II, verificou-se que aquelas crianças que obtiveram escore normal tiveram médias mais altas do que aquelas com resultado questionável; desse modo, o Teste $U$ de Mann Whitney indicou a presença de itens estatisticamente significativos, destacando-se define 3 objetos pelo uso $(0.82 \pm 0.39$, 
$p<0.01)$, define 5 palavras $(0.82 \pm 0.39, p<0.01)$, compreende 3 adjetivos $(0.75 \pm 0.44, p<0.01)$, conta 1 bloco $(0.86 \pm 0.36$, $p<0.05)$ e nomeia 4 cores $(0.79 \pm 0.42, p<0.05)$ (Tabela 1$)$.

Tabela 1. Média das pontuações dos itens avaliados pelo Teste de Triagem de Desenvolvimento de Denver II.

\begin{tabular}{|c|c|c|c|c|c|}
\hline \multirow{3}{*}{ Itens } & \multicolumn{4}{|c|}{ Desfecho Denver II } & \multirow{3}{*}{$p$ valor } \\
\hline & \multicolumn{2}{|c|}{ Suspeito } & \multicolumn{2}{|c|}{ Normal } & \\
\hline & Média & Desvio padrão & Média & Desvio padrão & \\
\hline Reconhece 2 ações & 0.96 & 0.19 & 1.00 & 00 & 0.27 \\
\hline Compreende 2 adjetivos & 1.00 & 00 & 1.00 & 00 & 1.00 \\
\hline Nomeia 1 cor & 1.00 & 00 & 1.00 & 00 & 1.00 \\
\hline Define 2 objetos & 0.96 & 0.19 & 1.00 & 00 & 0.27 \\
\hline Conta 1 bloco & 0.86 & 0.36 & 1.00 & 00 & $0.02^{*}$ \\
\hline Define 3 objetos pelo uso & 0.82 & 0.39 & 1.00 & 00 & $0.01 * *$ \\
\hline Compreende 4 preposições & 0.93 & 0.26 & 1.00 & 00 & 0.11 \\
\hline Nomeia 4 cores & 0.79 & 0.42 & 0.97 & 0.17 & $0.02 *$ \\
\hline Define 5 palavras & 0.82 & 0.39 & 1.00 & 00 & $0.01 * *$ \\
\hline Compreende 3 adjetivos & 0.75 & 0.44 & 0.97 & 0.17 & $0.01 * *$ \\
\hline Define 7 palavras & 0.86 & 0.36 & 1.00 & 00 & $0.02 *$ \\
\hline
\end{tabular}

Teste U: Teste não-paramétrico de Mann Whitney **p $<0.01 * p<0.05$ Fonte: Autores (2021).

A Tabela 2 mostra a associação do tipo de gênero com o desfecho do Denver II. Observou-se que as meninas obtiveram maiores médias de acertos nos seguintes itens: compreende 3 adjetivos $(0.94 \pm 0.25)$, reconhece 4 ações $(0.94 \pm$ $0.25)$, define 3 objetos pelo uso $(0.97 \pm 0.18)$ e conta 5 blocos $(0.91 \pm 0.30)$. Já os meninos destacaram-se nos itens reconhece 2 ações $(1.00 \pm 0)$, define 2 objetos $(1.00 \pm 0)$ e faz 2 analogias $(0.73 \pm 0.45)$. E ambos obtiveram as mesmas médias nos itens compreende 2 adjetivos $(1.00 \pm 0)$ e nomeia 1 cor $(1.00 \pm 0)$. 
Tabela 2. Relação entre o gênero e o desfecho do Teste de Triagem de Desenvolvimento de Denver II.

\begin{tabular}{|c|c|c|c|c|}
\hline \multirow{3}{*}{ Itens } & \multicolumn{4}{|c|}{ Sexo } \\
\hline & \multicolumn{2}{|c|}{ Feminino } & \multicolumn{2}{|c|}{ Masculino } \\
\hline & Média & Desvio-padrão & Média & Desvio-padrão \\
\hline Reconhece 2 ações & 0.97 & 0.18 & 1.00 & 0 \\
\hline Compreende 2 adjetivos & 1.00 & 0 & 1.00 & 0 \\
\hline Nomeia 1 cor & 1.00 & 0 & 1.00 & 0 \\
\hline Define 2 objetos & 0.97 & 0.18 & 1.00 & 0 \\
\hline Conta 1 bloco & 0.97 & 0.18 & 0.90 & 0.31 \\
\hline Define 3 objetos pelo uso & 0.97 & 0.18 & 0.87 & 0.35 \\
\hline Reconhece 4 ações & 0.94 & 0.25 & 0.83 & 0.38 \\
\hline Fala inteligível & 0.94 & 0.25 & 0.87 & 0.35 \\
\hline Compreende 4 preposições & 1.00 & 0 & 0.93 & 0.25 \\
\hline Nomeia 4 cores & 0.91 & 0.30 & 0.87 & 0.35 \\
\hline Define 5 palavras & 0.94 & 0.25 & 0.90 & 0.31 \\
\hline Compreende 3 adjetivos & 0.94 & 0.25 & 0.80 & 0.41 \\
\hline Conta 5 blocos & 0.91 & 0.30 & 0.83 & 0.38 \\
\hline Faz analogias -2 & 0.69 & 0.47 & 0.73 & 0.45 \\
\hline Define 7 palavras & 0.97 & 0.18 & 0.90 & 0.31 \\
\hline
\end{tabular}

Teste U: teste não-paramétrico de Mann Whitney

Fonte: Autores (2021)

\section{Discussão}

Nosso estudo apresentou uma amostra expressiva de crianças com desfecho questionável no desenvolvimento linguístico, mesmo sendo porcentagem inferior ao desfecho normal. Esse resultado foi similar também em pesquisas realizadas em outras regiões brasileiras, como no estudo de Simão, Sá e Cardoso (2017) em uma creche filantrópica em Fortaleza (CE), que encontraram 35,70\% das crianças com desempenho questionável na área da linguagem e enfatizaram a importância em discutir a respeito da temática, principalmente em relação aquelas que vivem em situações de vulnerabilidade. Outra pesquisa realizada no município de Matinhos (PR) relatou que 58.89\% de crianças apresentaram desempenho questionável na área da linguagem e foi verificado nítida associação entre o desempenho linguístico e fatores extrínsecos e intrínsecos, como o estado nutricional e o perfil cognitivo/emocional das crianças.

Outros resultados destacam-se em nossa pesquisa, como a diferença no desfecho no desenvolvimento linguístico em relação ao gênero da criança. As meninas obtiveram médias, em cada item individual, superiores aos meninos na maioria das tarefas do Denver II. Corroborando aos nossos resultados, um estudo realizado em uma Unidade de Educação Infantil (UEI) no 
município de Belém (PA) mostrou que as meninas também possuíam melhor desempenho neuropsicomotor, principalmente nas áreas da linguagem, motor fino-adaptativo e pessoal-social (Silva, Cavalcante, Heumann, \& Lima, 2018). A distinção anatômica, psíquica e emocional entre os gêneros é excepcionalmente complexa e permeia fenômenos biológicos, referentes à genética e à ação dos esteroides sexuais, e fenômenos socioambientais e comportamentais (Pletzer, Petasis, Ortner, \& Cahill, 2015; Araújo, Quadros, Murata, \& Israel, 2019).

Acredita-se também que essa diferença pode estar relacionada a fatores neurobiológicos. O processo de mielinização do córtex cerebral em meninas é mais precoce, fazendo com que áreas cerebrais, como área de Broca e de Wernicke, responsáveis pela propagação e associação da fala, sejam mais rapidamente desenvolvidas (Silva, Cavalcante, Heumann, \& Lima, 2018). Outro estudo baseado na técnica de Imagiologia por Tensor de Difusão realizado em indivíduo de 8 a 22 anos estabeleceu que o corpo caloso do sexo feminino é mais volumoso em comparação ao masculino, definindo que as meninas são melhores na comunicação inter-hemisférica, relacionada a processos comunicativos, analíticos e intuitivos; em contrapartida, os meninos têm melhor comunicação intra-hemisférica, sugerindo que são estruturados para facilitar a conectividade entre a percepção e a ação coordenada (Ingalhalikar et al., 2014; Reverter-Bañón, \& Medina-Vicent, 2020).

Todavia, outra pesquisa realizada em crianças com implante coclear assistidas pelo Serviço de Otorrinolaringologia do Centro Hospitalar e Universitário de Coimbra, Portugal, teve resultados controversos em comparação aos estudos supracitados, pois nesta pesquisa, apesar da baixa significância estatística, o sexo masculino obteve desempenho melhor durante a realização do projeto (Ramos, Jorge, Teixeira, Ribeiro, \& Paiva, 2015).

Baseado no exposto acima, observam-se resultados divergentes. Autores opõem-se ao tentar explicar e justificar a influência do gênero no desempenho neuropsicomotor. É possível que estas diferenças não estejam associadas apenas no atributo de natureza neurobiológica, mas às concepções sociais que são atribuídas a cada gênero e a educação historicamente diferenciada em detrimento às expectativas sociais e a natureza das atividades lúdicas, como jogos e brincadeiras (Silva, Cavalcante, Heumann, \& Lima, 2018). As meninas são, desde a mais tenra idade, estimuladas a realizarem brincadeiras sociais e simbólicas, como faz-de-conta, e os meninos a motricidade ampla (Gregovisk, Silva, \& Hlavac, 2016).

Nesse contexto, uma pesquisa realizada em uma UEI no município de Belém (PA) concluiu que brincadeiras e jogos influenciam no desenvolvimento infantil. As crianças do sexo feminino apresentaram melhor desempenho nas áreas de desenvolvimento relacionados a linguagem, motor-fino adaptativo e pessoal-social (Teixeira, Lourenço, Costa, Oliveira, \& Cruz, 2019). Contudo, o estudo realizado no município de Recife (PE) mostrou melhor desempenho da motricidade ampla nos meninos (Oliveira, Oliveira \& Cattuzzo, 2013). Isso mostra que os papeis sociais estabelecidos para cada gênero é refletido nas atividades lúdicas das crianças. É notória a importância em desenvolver brincadeiras que possam estimular todos os campos do desenvolvimento e promover um ambiente lúdico adequado, para que haja um positivo catalizador na aquisição da fala dessas crianças, bem como para as demais áreas (Gregovisk, Silva, \& Hlavac, 2016).

\section{Considerações Finais}

A análise dos resultados da pesquisa permitiu compreender aspectos importantes acerca do desenvolvimento linguístico e a diferença entre gêneros em crianças de uma creche pública. Os valores demonstram que as meninas tiveram melhor desempenho que meninos, resultados que, conforme literaturas, podem ser em detrimento, majoritariamente, dos estímulos neurobiológicos, ambientais e sociais.

Ao verificar o desenvolvimento infantil em uma visão integral nas quais essas crianças vivem, nossos desfechos sugerem que, embora as intervenções focadas no amparo individual, como pleno acesso à saúde e medidas escolares possibilitem emanar melhores trajetórias de desenvolvimento, o maior efeito no processo linguístico pode provir de intervenções e políticas que apoiam o ambiente ecológico e familiar em que as crianças estão inseridas. Além disso, essa 
temática vem sendo fortemente discutida, a partir de argumentos que incluem os efeitos sobre o desenvolvimento cognitivo e social, processo de escolaridade, vulnerabilidades e risco de exclusão social; afirmando fortemente a necessidade de haver pesquisas na área, principalmente estudos de coorte, que utilizem outros instrumentos de análise da linguagem e demais fatores que interferem no desenvolvimento infantil.

Nesse ínterim, as preferências e comportamentos são idealizados e construídos, e a sociedade tende a perpetuar padrões de comportamento que consideram certo ou errado, aceitável ou passível de rejeição e estabelecendo um papel para cada gênero, inserindo as crianças, desde pequenas, neste contexto e fazendo-as seguirem esses padrões de comportamento que limitam o seu desenvolvimento. Deste modo, aponta-se a necessidade de orientar os pais e educadores, por meio de políticas públicas, cartilhas e manuais que possam instruí-los a estimular o desenvolvimento infantil de forma íntegra e rompendo estes paradigmas de gênero, além de oferecer subsídios adequados para o impoluto estímulo ao desenvolvimento de todas as habilidades da criança.

Ademais, nessa pesquisa, apesar dos importantes resultados, houveram limitações referente ao tipo de estudo escolhido, pois este se restringe a coletas em um único momento e não há um acompanhamento evolucional dessas crianças, mostrando a relevância em estimular estudos de coorte e que tenham outras variáveis pertinentes para o estudo, como o ambiente familiar e o escolar.

\section{Referências}

Araújo, L. B., Quadros, D. A., Murata, M. P. F., \& Israel, V. L. (2019). Avaliação do desenvolvimento neuropsicomotor de crianças de 0 a 5 anos em centros de educação pública infantil. Revista CEFAC, 21(3), 1-9. http://dx.doi.org/10.1590/1982-0216/201921312918

Ciribelli, C. J., \& Rasera, E. F. (2019). Construções de Sentido sobre a Diversidade Sexual: Outro Olhar para a Educação Infantil. Psicologia: Ciência e Profissão,

39, 1-15. https://doi.org/10.1590/1982-3703003175599

Costa, E. F., Cavalcante, L. I., \& Dell'aglio, D. D. (2015). Perfil do Desenvolvimento da Linguagem de Crianças, segundo o Teste de Triagem de Denver II. Revista CEFAC, 17(4), 1090-1102. http://dx.doi.org/10.1590/1982-0216201517418514

Costa, P., Palombo, C. N., Silva, L. S., Silva, M. T., Mateus, L. V., \& Buchhorn, S. M. (2019). Ações de extensão universitária para translação do conhecimento

sobre desenvolvimento infantil em creches: Relato de experiência. Revista de Enfermagem da USP, 53, 1-8.

http://dx.doi.org/10.1590/S1980220X2018020603484

Duarte, N., Vasconcelos, M. A., \& Batalhas, I. (2011). Alterações adquiridas da linguagem na infância. Revista da Sociedade Portuguesa de Medicina Física e de Reabilitação, 20(1), 45-50. http://dx.doi.org/10.25759/spmfr.7

Eliot, L. (2013). Cérebro azul ou rosa: O impacto das diferenças de gênero na educação: Penso.

Gregoviski, V. R., Silva, F. L. L., \& Hlavac, L. A. B. (2016). “É Menino ou Menina?': A Construção da Identidade de Gênero Através dos Brinquedos”. Perspectiva, 40(152), 89-99. http://www.uricer.edu.br/site/pdfs/perspectiva/152_597.pdf

Guerim, L. D. (2020). Neurociência localizada: Revendo diferenças de sexo/gênero em pesquisas sobre o cérebro. Veritas, 65(2), 1-10. http://dx.doi.org/10.15448/1984-6746.2020.2.36565

Ingalhalikar, M., et al. (2014). Sex differences in the structural connectome of the human brain. PNAS, 111(2), 823-828. https://doi.org/10.1073/pnas.1316909110

Li, P., Legault, J., \& Litcofsky, K. A. (2014). Neuroplasticity as a function of second language learning: Anatomical changes in the human brain. Elsevier, 58, 301-324. http://dx.doi.org/10.1016/j.cortex.2014.05.001

Longo, I. A., Tupinelli, G. G., Hermógenes, C., Ferreira, L. V., \& Molini-Avejonas, D. R. (2017). Prevalência de alterações fonoaudiológicas na infância na região oeste de São Paulo. Communication Disorders, Audiology and Swallowing, 29(6), 1-7. http://dx.doi.org/10.1590/2317-1782/20172016036

Mélo, T. R., Lucchesi, V. O., Junior, E. J., \& Signorelli, M. C. (2020). Caracterização do desenvolvimento neuropsicomotor e de linguagem de crianças atendidas

por grupos no Núcleo Ampliado de Saúde da Família: Uma abordagem interprofissional. Revista CEFAC, 22(3), 1-10. http://dx.doi.org/10.1590/1982$0216 / 202022314919$

Oliveira, D. S., Oliveira, I. S., \& Cattuzzo, M. T. (2013). A influência do gênero e idade no desempenho das habilidades locomotoras de crianças de primeira infância. Revista Brasileira de Educação Física e Esporte, 27(4), 47-55. https://www.scielo.br/pdf/rbefe/v27n4/v27n4a12.pdf 
Research, Society and Development, v. 10, n. 7, e36410716214, 2021

(CC BY 4.0) | ISSN 2525-3409 | DOI: http://dx.doi.org/10.33448/rsd-v10i7.16214

Passaglio, N. J., Souza, M. A., Souza, V. C., Scopel, R. R., \& Lemos, S. M. (2015). Perfil fonológico e lexical: Interrelação com fatores ambientais. Revista CEFAC, 17(4), 1071-1078. http://dx.doi.org/10.1590/1982-0216201517419813

Pinheiro, M. (2007). Fundamentos de Neuropsicologia: O desenvolvimento cerebral da criança. Revista Vita et Sanitas, 1(1), 1-15.

Pinto, F. C., Isotani, S. M., Sabatés, A. L., \& Perissinoto, J. (2015). Denver II: Comportamentos propostos comparados aos de crianças paulistanas. Revista CEFAC, 17(4), 1262-1269. https://doi.org/10.1590/1982-0216201517418214

Pletzer, B., Petasis, O., Ortner, T. O \& Cahill, L. (2015). Interactive effects of culture and sex hormones on the sex role self-concept. Frontiers in Neuroscience,

9(240). https://doi.org/10.3389/fnins.2015.00240

Raffaini, P. T. (2018). As meninas são de pano e os meninos são de chumbo? Cultura material e literatura. Secuencia, n.spe, 177-187. https://doi.org/10.18234/secuencia.v0i0.1665

Ramos, D., Jorge, J. X., Teixeira, A., Ribeiro, C., \& Paiva, A. (2015). Desenvolvimento da linguagem em crianças com implante coclear: Terá o gênero alguma

influência?. Revista CEFAC, 17(2), 535-541. http://dx.doi.org/10.1590/1982-021620155214

Reverter-Bañón, S., \& Medina-Vicent, M. (2020). La diferencia sexual em las neurociências y la neuroeducación. Crítica, 50(150), 3-26. http://dx.doi.org/10.22201/iifs.18704905e.2018.13

Silva, M. L., Cavalcante, L. I. C., Heumann, S., \& Lima, T. V. R. (2018). Relação entre gênero e desempenho neuropsicomotor de crianças em Belém, Brasil. Ciência \& Saúde Coletiva, 23(8), 2721-2730. http://dx.doi.org/10.1590/1413-81232018238.13202016

Simão, A. L., Sá, F. E., \& Cardoso, K. V. (2017). Aplicação da escala de Denver II em crianças assistidas em uma creche filantrópica de Fortaleza. Repositório

Institucional da Universidade Federal do Ceará. http://www.repositorio.ufc.br/handle/riufc/33252

Teixeira, N. M. P., Lourenço, P. B., Costa, E. F., Oliveira, L. S. M., \& Cruz, D. A. (2019). Desenvolvimento neuropsicomotor e o brincar de crianças em uma unidade de educação infantil. Revista de Terapia Ocupacional da Universidade de São Paulo, 30(2), 116-123. https://doi.org/10.11606/issn.22386149.v30i2p116-123

Wermke, K., Quast, A., \& Hesse, V. (2018). From melody to words: The role of sex hormones in early language development. Hormones and Behavior, 104, 206-215. https://doi.org/10.1016/ j.yhbeh.2018.03.008

Zauche, L. H., Thul, T. A., Mahoney, A. E., \& Stapel-Wax, J. L. (2016). Influence of language nutrition on children's language and cognitive development: An

integrated review. Early Childhood Research Quarterly, 36, 318-333. https://doi.org/10.1016/j.ecresq.2016.01.015 